\title{
Making Sense of Singular Gauge Transformations in $1+1$ and $2+1$ Fermion Models
}

\author{
C.D. Fosco* \\ Centro Atómico Bariloche \\ 8400 Bariloche, Argentina \\ F.A. Schaposnik ${ }^{\dagger}$ \\ Departamento de Física, Universidad Nacional de La Plata \\ C.C. 67, (1900) La Plata, Argentina
}

April 3, 2021

\begin{abstract}
We study the problem of decoupling fermion fields in $1+1$ and $2+1$ dimensions, in interaction with a gauge field, by performing local transformations of the fermions in the functional integral. This could always be done if singular (large) gauge transformations were allowed, since any gauge field configuration may be represented as a singular pure gauge field. However, the effect of a singular gauge transformation of the fermions is equivalent to the one of a regular transformation with a non-trivial action on the spinorial indices. For example, in the two dimensional case, singular gauge transformations lead naturally to chiral transformations, and hence to the usual decoupling mechanism based on Fujikawa Jacobians. In $2+1$ dimensions, using the same procedure, different transformations emerge, which
\end{abstract}

*Investigador CONICET

$\dagger$ Investigador CICBA 
also give rise to Fujikawa Jacobians. We apply this idea to obtain the v.e.v of the fermionic current in a background field, in terms of the Jacobian for an infinitesimal decoupling transformation, finding the parity violating result. 


\section{Introduction}

We shall be concerned with $\mathcal{Z}(A)$, the generating functional for massless fermions in the presence of an external gauge field $A_{\mu}$, in $D$ (Euclidean) spacetime dimensions:

$$
\mathcal{Z}[A]=\int \mathcal{D} \bar{\psi} \mathcal{D} \psi \exp \left[-\int d^{D} x \bar{\psi}(\not \partial+i \not A) \psi\right]
$$

for the cases $D=2$ and $D=3$.

We start from the observation that any non-trivial, regular, external gauge field coupled to fermions can always be written in terms of a pure gauge field. The price to be paid is that the gauge field transformation associated with this pure gauge field is necessarily a singular one. It is then possible to perform a singular gauge transformation rendering the fermion action free. The procedure is of course formal, in the sense that singularities in the fermionic transformations make complicated to check whether the new variables are strictly free and, moreover, whether they induce a non-trivial Jacobian at the quantum level.

These are indeed the reasons why these transformations cannot be naively used to decouple fermions from an external gauge field. We shall see, however, that there is a way to make sense of them. The clue for this, lies in the observation that a singular gauge transformation for the fermions can be written in terms of a regular transformation that is not the same for all the spinor components. Namely, this regular transformation does not change all components of the spinor with the same phase factor and can also mix different components.

To understand the statements above, consider the case of 2 space-time dimensions. It is well-known in this case that a local regular chiral transformation decouples massless fermions from the gauge field 1 . When performed as a path-integral change of variables, the decoupling chiral transformation leads to a free fermionic action but has an associated non-trivial Jacobian [1]. Its calculation [2] allows one to have a complete control of the decoupled theory at the quantum level. What is less known is that there is an alternative way of decoupling two-dimensional massless fermions and this through a singular gauge transformation. As we shall see, one can find a connection between both transformations, the regular chiral one and the singular

\footnotetext{
${ }^{1}$ By "local" we mean that the transformation relates fermionic fields at the same spacetime point.
} 
gauge one. Chiral transformations suffer from the well-known anomaly phenomenon, an effect of far-reaching consequences [3]. These effects manifest themselves both in the operatorial and path-integral versions of the theory.

Concerning the path-integral framework, since the transformed action is free, a non-trivial Jacobian taking into account quantum effects is to be expected and it should coincide with the chiral Jacobian since both transformations left us with the same free action.

This two-dimensional example shows that either transformation, one that changes all spinor components with the same (singular) phase and the other changing each component with regular phases differing in signs, can be used to completely decouple massless fermions from an external gauge field.

Once one is convinced that at least in $D=2$ dimensions the two approaches are equivalent, however different their derivations seem to be, one can try to make sense of singular transformations for $D=3$ and to determine what kind of equivalent regular transformation emerges. This is the main purpose of the present work. We shall see that in $D=3$ dimensions, it is possible to obtain non perturbative information through the connection of infinitesimal singular gauge transformations with regular ones, whose Jacobian can be calculated. In particular, using the procedure described above, we compute the v.e.v. of the fermion current in an Abelian gauge field background in $D=3$, finding the well-known parity violating result [4]- [5]. In the present case the parity anomaly stems from the non-triviality of a Fujikawa Jacobian as it happens in even dimensions with the axial anomaly and the chiral Jacobian.

The plan of the paper is as follows: we first discuss in section 2 the twodimensional case in which the connection between singular gauge and regular chiral transformations is transparent. Inspired by this example, we discuss in section 3 the three dimensional case, computing the Fujikawa Jacobian for an infinitesimal regular transformation. We then apply this result to the evaluation of the v.e.v. of the fermion current in the presence of an external field.

\section{The Abelian case in $D=2$}

This is of course the simplest case we may consider. Since, for massless fermions, the fermionic determinant may be exactly calculated, everything may be checked on the safe ground of the exact solutions. The first step in the 
construction of the large gauge transformations is similar to one used in the chiral transformation approach, namely, the decomposition of the external gauge field $A_{\mu}$ into the sum of a gradient of a scalar $\varphi$ plus the curl of a pseudoscalar $\sigma$ :

$$
A_{\mu}=\partial_{\mu} \varphi+\epsilon_{\mu \nu} \partial_{\nu} \sigma
$$

Of course, the only pure gauge part of $A_{\mu}$ is, at this point, the gradient term. We can however always force the curl term to also look as a pure gauge, if we define another scalar field $\rho$, such that

$$
\epsilon_{\mu \nu} \partial_{\nu} \sigma=\partial_{\mu} \rho
$$

Taking the curl on both sides of (3), we see that

$$
\epsilon_{\mu \nu} \partial_{\mu} \partial_{\nu} \rho=-\partial^{2} \sigma=F,
$$

where $F(x) \equiv \epsilon_{\mu \nu} \partial_{\mu} A_{\nu}(x)$.

Thus, the partial derivatives do not commute when acting on $\rho$, except in the trivial case $F=0$. We shall also see that the singularity is of the branching point type, namely, $\rho$ is a sort of angular variable (see (17) below). Note that the singularities are in $\rho$, not on $\partial_{\mu} \rho$ which is the gauge invariant part of the gauge field, and for which we shall assume a trivial topology.

By using (2) and (3), the fermionic action may be written as

$$
S_{F}=\int d^{2} x \bar{\psi}[\not \partial+i \not \partial(\varphi+\rho)] \psi
$$

where the fermions are coupled just to a pure gauge. This suggests the introduction of the singular (because of $\rho$ ) decoupling transformations

$$
\begin{aligned}
& \psi(x)=\exp [-i(\varphi(x)+\rho(x))] \chi(x) \\
& \bar{\psi}(x)=\bar{\chi}(x) \exp [i(\varphi(x)+\rho(x))],
\end{aligned}
$$

which are the transformations we want to make sense of. Of course we will ignore the $\varphi$-dependent part, since it is regular and produces no anomalous Jacobian (in an invariant regularization, which we shall assume). To understand the nature of the singularity, let us consider again equation (3), defining $\rho$. Integrating both sides along a given curve $\mathcal{C}$, with origin at a fixed point $P$ of coordinates $x_{P}$, and end at the point of coordinates $x$, we see that

$$
\rho(x)-\rho\left(x_{P}\right)=\int_{x_{P}}^{x} d \xi_{\mu} \epsilon_{\mu \nu} \partial_{\nu} \sigma .
$$


Of course, the value of $\rho(x)$ will be, in general, dependent on both $P$ and $\mathcal{C}$. We shall put the point $P$ at infinity.

In order to avoid the existence of zero modes for the Dirac operator, we shall assume that

$$
\oint_{R \rightarrow \infty} d \xi_{\mu} \epsilon_{\mu \nu} \partial_{\nu} \sigma=0
$$

holds. (The suffix $R \rightarrow \infty$ means that the circulation is to be calculated along a circle of infinite radius). Indeed, because of eq,(2), eq.(8) is equivalent to

$$
\oint_{R \rightarrow \infty} d \xi_{\mu} A_{\mu}=0
$$

and, by Stokes theorem, this is tantamount to requiring the net magnetic flux to vanish

$$
\int d^{2} x \epsilon_{\mu \nu} \partial_{\mu} A_{\nu}=0
$$

A non-zero total flux would imply the existence of zero modes, and the vanishing of the determinant of the Dirac operator in such a background. For a non-trivial (although topologically trivial) $A_{\mu}$, the function $\rho$ will have singularities, since the circulation of $A_{\mu}$ along at least one finite closed curve will be non-zero.

That the transformations (6) are not justified should be self-evident: the new fermionic fields will have singularitites introduced by the transformation, which then takes the original fields out of the initial space. To make sense of these transformations, we first consider an infinitesimal version of the singular part of (6)

$$
\begin{aligned}
& \delta \psi(x)=-i \eta \rho(x) \psi(x) \\
& \delta \bar{\psi}(x)=i \eta \bar{\psi}(x) \rho(x)
\end{aligned}
$$

where $\eta$ is an infinitesimal parameter.

It is important to realize at this point that in the action (5) it is not $\rho$ but $\not \rho$ what appears, and this particular combination is regular. To see this, we just need to use the identity

$$
\not \partial\left[\rho(x)+i \gamma_{5} \sigma(x)\right]=0
$$

which is a consequence of the two dimensional Dirac algebra (we shall see however that a similar relation exists in $D=3$ ). We want to remark that (12) relates the (regular) derivatives of $\rho$ to the (also regular) derivatives of $\sigma$. 
Then one realizes that, because of (12), the effect of a chiral transformation is equivalent to the effect of a non-chiral large gauge transformation. (Note that we are not saying that the singular field $\rho$ is somehow transformed into a regular field by multiplying it by $\gamma_{5}$ ).

In this way, and just by the use of (12), one arrives to an equivalent representation of the action (河),

$$
S_{F}=\int d^{2} x \bar{\psi}\left[\not \partial+i \not \partial \varphi+\not \partial \gamma_{5} \sigma\right] \psi
$$

which is of course the starting point of the usual decoupling by a chiral transformation. We may then forget the singular transformation induced by $\rho$ in favour of the regular one generated by $-i \gamma_{5} \sigma$, which is a chiral transformation. They may differ at most in a transformation generated by $\xi$, a solution of the free equation

$$
\not \partial \xi=0 .
$$

Equation (14) implies that each component of $\xi$ has to be either analytic or anti-analytic on the plane. Thus they are constants. We have learnt that the only thing we could miss by replacing the vector singular transformation by the chiral regular one is the Jacobian due to a general global chiral transformation: This is a $\theta$-vacua term, which is zero because of (10).

The regular infinitesimal transformations (corresponding to the singular ones, eq.(111), are

$$
\begin{aligned}
& \delta \psi(x)=-\eta \sigma(x) \gamma_{5} \psi(x) \\
& \delta \bar{\psi}(x)=-\eta \bar{\psi}(x) \gamma_{5} \sigma(x) .
\end{aligned}
$$

Once one obtains this formula for the regular transformation, one proceeds in the usual way to derive the Jacobian, which will of course need regularization. Note that the parameter of the transformation is actually the same one uses when dealing with the standard procedure involving anomalous chiral Jacobians [1].

We conclude this section by presenting the explicit form of the fields $\sigma$ and $\rho$, since in two dimensions their expressions are particularly simple and illuminating ]. For $\sigma$, we have

$$
\sigma(x)=-\frac{1}{2 \pi} \int d^{2} y \ln [\mu|x-y|] F(y)
$$

\footnotetext{
${ }^{2}$ Similar relations appear in different contexts, see for example [6].
} 
where $\mu$ is a constant with the dimensions of a mass. Regarding $\rho$, it is also immediate to see that

$$
\rho(x)=\frac{1}{2 \pi} \int d^{2} y \Theta(x-y) F(y)
$$

where $\Theta(x)=\arg \left(\frac{x_{2}}{x_{1}}\right)$. It is obvious that $\rho$ has discontinuities, associated with the branching point of the angle function $\Theta$, while $\sigma$ does not suffer from those singularities. Equations (17) and (16) can be collected into a single matrix equation

$$
-\sigma(x)+i \gamma_{5} \rho(x)=\frac{1}{2 \pi} \int d^{2} y \ln \left[x_{1}-y_{1}+i \gamma_{5}\left(x_{2}-y_{2}\right)\right] F(y) .
$$

Either from these relations, or even from (3), we may see that $\rho$ and $\sigma$ satisfy the Cauchy-Riemann equations, and are then the real and imaginary parts of an analytic function:

$$
f=\rho+i \sigma, \frac{\partial}{\partial \bar{z}} f=0
$$

We may rephrase the equivalence between regular chiral and large non chiral transformations by saying that the effect of a gauge transformation generated by $\rho$ is equivalent to the one of a chiral transformation generated by the dual of $\rho$, which is $\sigma$. The term "dual" is understood here in the sense that vortex-like configurations (remember $\rho$ is angular), are transformed into "Coulomb" configurations (i.e., the potential of a charge distribution).

Of course, this picture also holds the other way around: a singular chiral pure gauge field is equivalent to a regular non-chiral pure gauge field, and thus it can be gauged away! The fermions are free in this case. The reason for the "asymmetry" between these case and the original one is that one usually regulates the Jacobian using the operator $\not D$. A more general choice would put both cases on a similar footing.

\section{The Abelian case in $d=3$}

We consider again the generating functional (1), but for the $D=3$ case. Now the (Hermitian) $\gamma$ matrices satisfy the relations

$$
\gamma_{\mu} \gamma_{\nu}=\delta_{\mu \nu} I+i \epsilon_{\mu \nu \lambda} \gamma_{\lambda}
$$


where $I$ denotes the identity matrix.

The decomposition of $A_{\mu}$ is now slightly different, since a general $A$ configuration will have three independent components. We shall use a scalar field $\varphi$ and a pseudovector $\sigma_{\mu}$,

$$
A_{\mu}=\partial_{\mu} \varphi+\epsilon_{\mu \nu \lambda} \partial_{\nu} \sigma_{\lambda}
$$

The decomposition (21) apparently includes $1+3=4$ components on the right hand side. Note, however, that $A_{\mu}$ is insensitive to the transformations

$$
\sigma_{\lambda} \rightarrow \sigma_{\lambda}+\partial_{\lambda} \alpha
$$

which reduce by one the actual number of components. We can in fact impose a gauge condition on $\sigma_{\mu}$, the natural choice being the Lorentz condition

$$
\partial \cdot \sigma=0
$$

which we shall adopt. We may again write the curl term in (21) as the gradient of a scalar field $\rho$,

$$
\epsilon_{\mu \nu \lambda} \partial_{\nu} \sigma_{\lambda}=\partial_{\mu} \rho
$$

Of course $\rho$ will have singularities

$$
\epsilon_{\lambda \mu \nu} \partial_{\mu} \partial_{\nu} \rho=\partial_{\mu} \partial \cdot \sigma-\partial^{2} \sigma_{\mu}=-\partial^{2} \sigma_{\lambda}=\tilde{F}_{\lambda}
$$

whenever $\tilde{F}_{\mu} \equiv \epsilon_{\mu \nu \lambda} \partial_{\nu} A_{\lambda} \neq 0$. Integrating along a curve starting at a point $P$ at infinity, we may write

$$
\rho(x)-\rho\left(x_{P}\right)=\int_{x_{P}}^{x} d \xi_{\mu} \epsilon_{\mu \nu \lambda} \partial_{\nu} \sigma_{\lambda} .
$$

The fermionic action would then be

$$
S_{F}=\int d^{3} x \bar{\psi}[\not \partial+i \not \partial(\varphi+\rho)] \psi
$$

where the fermions are coupled to a (singular) pure gauge field. The singular decoupling transformations are then formally the same as for the twodimensional case (6)

$$
\begin{aligned}
& \psi(x)=\exp [-i \rho(x)] \chi(x) \\
& \bar{\psi}(x)=\bar{\chi}(x) \exp [i \rho(x)] .
\end{aligned}
$$


We then introduce regular infinitesimal transformations in exactly the same way as in the two dimensional case. The three dimensional analog of (12)

$$
\not \partial[\rho(x)+i \not \phi(x)]=0 .
$$

Thus we are led to consider the infinitesimal regular transformations

$$
\begin{aligned}
& \delta \psi(x)=\delta \phi(x) \psi(x) \\
& \delta \bar{\psi}(x)=\bar{\psi}(x) \delta \phi(x) .
\end{aligned}
$$

which are the infinitesimal regular version of the decoupling singular transformations (28). In the present $D=3$ case, we shall only perform infinitesimal decoupling transformations, which will allow us to compute v.e.v. of fermion currents $j^{\mu}=i \delta Z[A] / \delta A_{\mu}$. We then need to consider a variation on $A_{\mu}$ in $\mathcal{Z}[A]$

$$
\mathcal{Z}[A+\delta A]=\int \mathcal{D} \bar{\psi} \mathcal{D} \psi \exp \left[-\int d^{D} x \bar{\psi}(\not \partial+i(\not A+\delta / A) \psi]\right.
$$

Now, an infinitesimal change of fermion fields like (30) can be used to eliminate $\delta A_{\mu}$ from the action. That change will, very likely, produce a non trivial Jacobian $\mathcal{J}_{\delta \sigma}[A, \delta A]$

$$
\mathcal{Z}[A+\delta A]=\mathcal{J}_{\delta \sigma}[A, \delta A] \mathcal{Z}[A]
$$

with

$$
\mathcal{J}_{\delta \sigma}[A, \delta A]=\exp \left(\int d^{3} x \delta A_{\mu} \mathcal{G}_{\mu}[A]\right)
$$

To obtain the connection between $\delta \sigma_{\mu}$ and $\delta A_{\mu}$, note that from the relation (21) between $\sigma_{\mu}$ and $A_{\lambda}$, we can write

$$
\delta A_{\mu}^{\perp}=\epsilon_{\mu \nu \lambda} \partial_{\nu} \delta \sigma_{\lambda}
$$

where $\perp$ denotes transverse components. We shall connect the Jacobian (33) associated with a general gauge field variation $\delta A_{\mu}$ (i.e. including longitudinal components) with that associated with $\delta A_{\mu}^{\perp}$,

$$
\mathcal{J}\left[A+\delta A^{\perp}\right]=\exp \left(\int d^{3} x \delta A_{\mu} \mathcal{A}_{\mu}[A]\right)
$$


To this end, note that $\delta A_{\mu}^{\perp}$ can be thought of as a $\delta A_{\mu}$ variation in a gauge where the corresponding longitudinal component is fixed to zero. Including the 3 possible different choices for that component gives then twice the general variation we were looking for. Thus, a factor $3 / 2$ should be included,

$$
\mathcal{G}_{\mu}[A]=\frac{3}{2} \mathcal{A}_{\mu}[A] .
$$

Let us now compute the anomalous Jacobian associated with transformations (30). Indeed, under those transformations, the anomalous Jacobian reflecting the change in the fermionic integration measure is

$$
\begin{aligned}
& \mathcal{J}_{\delta \sigma}=\mathcal{J}_{\psi} \mathcal{J}_{\bar{\psi}} \\
& \mathcal{D} \psi \rightarrow \mathcal{D} \psi \mathcal{J}_{\psi} \\
& \mathcal{D} \bar{\psi} \rightarrow \mathcal{D} \bar{\psi} \mathcal{J}_{\bar{\psi}} .
\end{aligned}
$$

The Jacobians $\mathcal{J}_{\psi}$ and $\mathcal{J}_{\bar{\psi}}$ may be evaluated, to the first order in $\delta \sigma_{\mu}$, as follows

$$
\begin{aligned}
\mathcal{J}_{\psi} & =\exp [-\operatorname{Tr} \delta \not]] \\
\mathcal{J}_{\bar{\psi}} & =\exp [-\operatorname{Tr} \delta \not]
\end{aligned}
$$

where the trace is both functional and over the Dirac indices. This trace, as it happens in the $D=2$ case, is ill-defined, and needs regularization. In general, we shall have

$$
[\operatorname{Tr} \delta \not]_{r e g}=\int d^{3} x \delta \sigma_{\mu}(x) \mathcal{A}_{\mu}^{r e g}(x)
$$

where

$$
\mathcal{A}_{\mu}^{\text {reg }}(x)=\operatorname{tr}\left[\left\langle x\left|\gamma_{\mu} f\left(\not D, \Lambda_{i}\right)\right| x\right\rangle\right]
$$

where $\Lambda_{i}(i=1,2, \ldots)$ are the Pauli-Villars regulator masses,$f$ is a regulating function (which tends to 1 for large values of $\Lambda_{i}$ and regulates UV divergences), and tr denotes the trace over Dirac indices. We shall choose a Pauli-Villars regulator of the form

$$
f\left(\not D, \Lambda_{i}\right)=\frac{\Lambda_{1} \Lambda_{2}}{\Lambda_{2}-\Lambda_{1}}\left(\frac{1}{\not D+\Lambda_{1}}-\frac{1}{\not D+\Lambda_{2}}\right)
$$


where two regulators $\Lambda_{i}(i=1,2)$ with same sign are enough to ensure that infinities are eliminated in the limit $\Lambda_{i} \rightarrow \infty$. Only one term in the trace survives in this limit,

$$
\mathcal{A}_{\mu}^{r e g}(x)= \pm \frac{i}{24 \pi} \partial^{2} \epsilon_{\mu \nu \lambda} \partial_{\nu} \sigma_{\lambda}+\mathcal{O}\left(\frac{1}{\Lambda}\right)
$$

where the \pm results from the (common) sign choice for the $\Lambda_{i}$ 's. This yields, for the Jacobian $\mathcal{J}_{\psi}$,

$$
\mathcal{J}_{\psi}=\exp \left( \pm \frac{i}{24 \pi} \int d^{3} x \delta \sigma_{\mu} \partial^{2} A_{\mu}\right)
$$

The result for $\mathcal{J}_{\bar{\psi}}$ is identical so that the total Jacobian reads

$$
\mathcal{J}_{\delta \sigma}=\exp \left( \pm \frac{i}{12 \pi} \int d^{3} x \delta \sigma_{\mu} \partial^{2} A_{\mu}\right)
$$

We then see that there is a non-trivial Jacobian associated with transformations (30), the regular and infinitesimal version of the singular gauge transformations (28). In this sense transformations (28) (or their regular counterparts (30) are anomalous. Using eqs.(33)-(36) we get from (44) the Jacobian associated with a $\delta A_{\mu}$ variation

$$
\mathcal{J}_{\delta A}=\exp \left( \pm \frac{i}{8 \pi} \int d^{3} x \epsilon_{\mu \nu \lambda} \delta A_{\mu} \partial_{\nu} A_{\lambda}\right)
$$

From this we compute the v.e.v. of the fermionic current in the presence of an external gauge field, obtaining

$$
j_{\mu}=\mp \frac{1}{8 \pi} \epsilon_{\mu \nu \lambda} \partial_{\nu} A_{\lambda}
$$

This result coincides with the one originally obtained by Redlich [四]- [5] for the parity violating part of the fermion current in a constant field strength background, using the Schwinger method for evaluating the Euler-Heisenberg effective action. In our calculation, valid for arbitrary field strength, $j_{\mu}$ arises from a Fujikawa Jacobian. It should be noted that our regularization prescription (eqs.(41)) neglects the parity conserving part (which is absent in [5] because a constant field strength is considered).

It is worth remarking that the decoupling procedure followed here differs from the one applied in [7], since in that reference the decoupling transformations are non-local. Moreover, those non-local transformations induce non-anomalous (but nevertheless non-trivial) Jacobians. 
Also note that, both in $1+1$ and $2+1$ dimensions, a mass term for the fermions is not invariant under a regular transformation, what makes it difficult to relate the effects of singular and regular transformations.

We conclude by stressing that both in $1+1$ and $2+1$ dimensions the method presented in this work follows exactly the same steps: one first writes a singular gauge transformation - a finite decoupling one in $1+1$ dimensions, an infinitesimal one in $2+1$ dimensions. Then, one finds a regular equivalent transformation on fermions which can be properly handled. Associated with these transformations there are, at the quantum level, anomalous Jacobians which, for the regular transformations, can be easily computed. The Jacobians, associated with the chiral anomaly in $1+1$ dimensions and the parity anomaly in $2+1$ dimensions, correctly describe quantum aspects in both cases. This provides an alternative way of understanding exactly soluble two dimensional fermion models but also of computing relevant v.e.v.'s in higher dimensions. In fact, the same procedure described here could be applied to the calculation of the v.e.v. of the axial current in $3+1$ dimensions (through a decoupling transformation analysis, which shall involve the chiral anomaly in $3+1$ dimensions), the v.e.v. of the fermion current in $4+1$ dimensions (related to the $4+1$ dimensional parity anomaly), etc, with the advantage that the procedure is systematic and relies on the calculation of anomalous Jacobians whose regularization is well-understood.

\section{Acknowledgements}

C.D.F. is supported by CONICET, Argentina. F.A.S. is supported by CICBA, Argentina This work is supported in part by grants from CICBA, CONICET (PIP 4330/96), ANPCYT (PICT 97/2285, 97/0053) and Fundación Antorchas, Argentina.

\section{References}

[1] K. Fujikawa, Phys. Rev. Lett. 42 (1979) 1195; Phys. Rev. D21 (1980) 2848.

[2] R. Roskies and F.A. Schaposnik, Phys. Rev. D23 (1981) 558. 
[3] R. Jackiw, Lectures on Current Algebras and its Applications (eds. S.B. Treiman, R. Jackiw and D. Gross), Princeton University Press, 1972.

[4] S. Deser, R. Jackiw and S. Templeton, Phys. Rev. Lett. 48 (1982) 975; Ann. of Phys. (N.Y.) 140 (1982) 372.

[5] A.N. Redlich, Phys. Rev. Lett. 52 (1984) 18; Phys. Rev. D29 (1984) 236.

[6] A. Kovner, Int. J. Mod. Phys. A5 (1990) 3999.

[7] C. D. Fosco and J. C. Le Guillou, Nucl. Phys. B535 (1998) 483. 